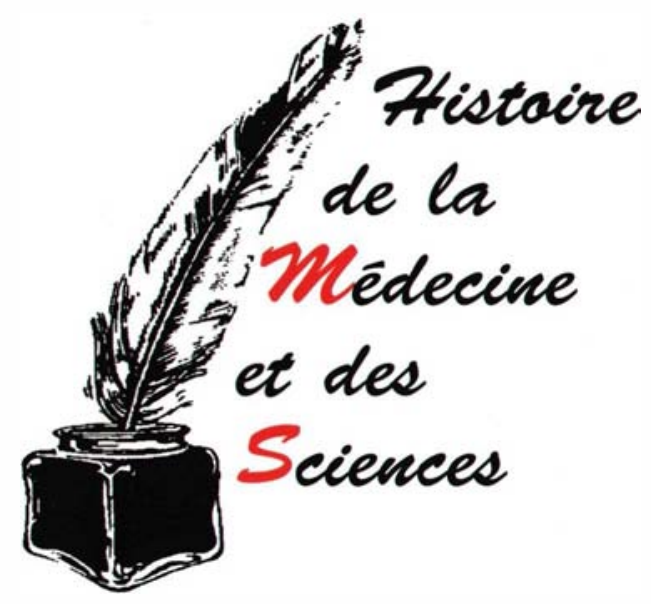

\title{
La transfusion sanguine, hier, aujourd'hui et demain
}

sion fut largement pratiquée et sauva de nombreux blessés.

Tout changea au début du siècle, lorsque Landsteiner découvrit l'existence des groupes sanguins de base. Il montra que les hématies peuvent porter des antigènes qu'il appela $\mathrm{A}$ et $\mathrm{B}$, ce qui donne lieu à quatre types sanguins (ou si l'on veut à quatre phénotypes): le groupe $\mathrm{A}$ ayant le facteur A, le groupe B caractérisé par la présence de $\mathrm{B}$, le groupe $\mathrm{O}$ dépourvu de l'un et de l'autre, enfin le groupe $A B$ qui véhicule les deux. Cette découverte fut rendue possible par le fait singulier que l'on rencontre de façon constante, spontanée, sans stimulation immunitaire, des anticorps sériques ne correspondant pas aux antigènes présents sur les hématies. On les appela pour cela agglutinines normales ou régulières. Les sujets $A$ ont un anti-B dans leur sérum, les sujets $B$ un anti-A, les sujets $A B$ rien, et les sujets $O$ les deux. Ainsi furent définies les «lois de compatibilité " qui obligeaient de choisir toujours un receveur dépourvu de tout anticorps capable d'hémolyser les hématies transfusées, sous peine de provoquer des accidents graves dont la découverte de Landsteiner avait révélé la cause.

Désormais les chocs transfusionnels étaient devenus très rares mais n'avaient pas complètement disparu. On en rencontrait parfois chez des polytransfusés qui appartenaient cependant au même groupe du système $\mathrm{ABO}$. Or, tous ces patients véhiculaient un "nouvel" anticorps, absent chez les sujets normaux, et qui identifiait un facteur inconnu jusque-là.

Entre 1937 et 1940, Landsteiner et son élève Wiener injectent au lapin des hématies de diverses espèces de singe dont Macacus rhesus (Macaca mulata) dans l'espoir de provoquer l'apparition d'immunanticorps qui reconnaîtraient des antigènes présents non seulement sur des hématies de singes donneurs, mais en même temps chez l'homme.

C'est ainsi qu'ils obtinrent un réactif capable d'agglutiner les hématies des macaques, mais pouvant agglutiner aussi les cellules rouges de $85 \%$ d'Américains blancs choisis au hasard, et dits $\mathrm{Rh}^{+}$mais demeurant sans action sur les $15 \%$ autres. Cela démontrait que le premier lot d'humains portait dans son sang le même facteur que celui présent chez tous les rhésus (ou tout au moins un facteur apparenté).

On étudia les cas d'accidents transfusionnels non expliqués. Il devint clair que dans la grande majorité des cas, on pouvait les attribuer au facteur rhésus. Un sujet rhésus négatif $\left(\mathrm{Rh}^{-}\right)$ recevant à plusieurs reprises du sang rhésus positif $\left(\mathrm{Rh}^{+}\right)$s'immunisait à cet antigène (apparition d'une agglutinine anti-Rh dite anticorps irrégulier) et, dès les transfusions suivantes accusait une hémolyse massive du sang injecté. Mais il arrivait aussi que la première transfusion soit fatale. L'explication en fut donnée en 1941 par Levine et ses collaborateurs, quand ils démontrèrent qu'une fem- transfuser. Au cours de la guerre franco-prussienne de 1870 , la transfu- 
me $\mathrm{Rh}^{-}$portant un fotus $\mathrm{Rh}^{+}$pouvait s'immuniser contre le facteur $\mathrm{Rh}$ à l'occasion du passage d'hématies fœtales à travers le placenta. Les anticorps ainsi formés peuvent à leur tour traverser le placenta dans le sens mère-enfant, se fixer sur les cellules du nouveau-né et déclencher chez lui une maladie hémolytique néonatale, avec anémie, ictère grave et dans quelques cas particulièrement sévères, léser un certain nombre de noyaux du système nerveux central: c'est l'ictère nucléaire, autrefois très redouté des accoucheurs.

En étudiant un grand nombre d'accidents obstétricaux ou transfusionnels, il apparut vite que le facteur Rh n'était pas toujours seul en cause, mais que d'autres antigènes apparentés (car hérités ensemble) pouvaient se trouver à l'origine d'accidents identiques. On identifia d'abord trois paires d'allèles baptisées par des lettres: $\mathrm{D} / \mathrm{d} ; \mathrm{C} / \mathrm{c} ; \mathrm{E} / \mathrm{e}$ correspondant en réalité à trois séries polyalélliques: D,DU ; C,CW ; CXc, CU ; E, EW, Et, EU, eS, e, etc., pour ne citer que les principaux. Ultérieurement, plus de quarante variantes de ce système devaient être décrites.

De plus, bien d'autres systèmes, indépendants du Rh responsables d'alloimmunisation post-transfusionnelle ou par incompatibilité fœeto-maternelle ont été découverts. Parmi les plus importants on peut citer Kell; Duffy; Kidd ; MNSs et ses «satellites " au nombre d'une dizaine.

Quel que soit le facteur responsable (dans la pratique surtout D du système $\mathrm{Rh}$ ), la maladie hémolytique néonatale est aujourd'hui bien maîtrisée. Au début, on surveillait l'apparition éventuelle, en cours de grossesse, d'immunanticorps anti-D chez toute mère $\mathrm{Rh}^{-}$de façon à pratiquer, dès la naissance (souvent provoquée) une exsanguino-transfusion chez le nouveau-né au moyen de sang $\mathrm{Rh}^{-}$, et donc invulnérable aux anticorps maternels. Cette thérapeutique donnait dans certains cas de bons résultats. Mais son efficacité n'était pas constante, l'agression des anticorps maternels contre le fotus ayant déjà provoqué des lésions irréversibles en fin de vie intra-utérine. Un nouveau pas fut franchi lorsque l'on sut pratiquer l'exsanguino-transfusion avant la venue au monde du petit patient. Les résultats ont été plus encourageants. Aujourd'hui, la thérapeutique est devenue très efficace. Elle consiste à injecter le facteur en cause, le plus souvent $\mathrm{Rh}^{+}$à la femme $\mathrm{Rh}^{-}$, de façon à neutraliser l'anticorps anti-Rh dès sa formation. La grossesse peut alors se dérouler normalement.

Pour la transfusion proprement dite, longtemps on a utilisé la technique du "bras à bras ", dans laquelle la veine du donneur était reliée directement à celle du receveur, le flux sanguin étant assuré par un système de pompage. Un progrès d'importance majeure fut réalisé au cours du premier conflit mondial, lorsque l'on découvrit que la coagulation pouvait être évitée en recueillant le sang dans des bouteilles contenant une petite quantité d'un mélange de dextrose, de citrate de soude et d'acide citrique. Désormais, il devenait possible de conserver plusieurs jours le sang au réfrigérateur après en avoir effectué le groupage. La transfusion au blessé se pratiquait dès le premier poste de secours, ce qui permettait de le maintenir en vie jusqu'à son arrivée dans un centre chirurgical. Entre les deux guerres, l'on apprend à séparer les cellules et le plasma. Ce dernier, une fois desséché, se conserve pendant des mois à la température ambiante, tout en gardant ses qualités physiologiques (en particulier pouvoir antichoc). Au moment de l'emploi, ce plasma sec est dilué dans de l'eau distillée et injecté aussitôt. Cette méthode sera largement utilisée par l'armée américaine du Pacifique, où le front est morcelé en un grand nombre d'îles, parfois distantes de milliers de kilomètres, ce qui rend impossible tout transport de sang complet.

La paix revenue, on entreprend de scinder plasma et cellules en leurs différents constituants. Les hématies sont réservées aux grands anémiques, les plaquettes aux malades qui saignent. Quant au plasma, trois de ses composantes vont entrer dans la thérapeutique courante: le facteur VIII appliqué à la majorité des hémophiles (incapables de le synthétiser), l'albumine, dont le rôle essentiel est antichoc (traumatisés, brûlés, dénu- tris, etc.) et les globulines, porteuses des molécules d'anticorps que l'on injectera aux grands infectés. On peut ainsi sous un volume réduit, apporter au malade exactement ce qui lui manque et rien d'autre. L'utilisation de sang total n'a pas disparu pour autant: l'indication majeure en est l'intervention chirurgicale, les accidents de la route ou du travail, et toutes les hémorragies massives.

Bien que sa pratique ait transformé la chirurgie et la médecine, la transfusion sanguine comporte des risques aujourd'hui bien connus. Certes, les accidents liés à des erreurs de groupage sont devenus exceptionnels (un sur un million). Cependant la transmission de germes pathogènes par le sang injecté doit toujours être redoutée. Longtemps, les seules maladies en cause, grand souci des transfuseurs, étaient la syphilis répartie un peu partout dans le monde, et le paludisme, fréquent chez les coloniaux; aujourd'hui, les deux sont facilement évités.

Après la Seconde Guerre mondiale, avec le développement des transports et leur rapidité, des viroses longtemps limitées à une zone géographique précise, ont quitté leur berceau ancestral pour se mondialiser. D'abord dans les années 1960 l'hépatite $\mathrm{B}$ (dite à la seringue par opposition à la $\mathrm{A}$, alimentaire) entraîna des accidents graves et parfois mortels. Sa transmission sexuelle fut aussi démontrée. Fort heureusement, un vaccin a été mis au point. Aujourd'hui, au moins dans les pays industrialisés, l'hépatite $\mathrm{B}$ a notablement diminué, et il n'est pas impossible que l'on arrive à l'éradiquer. On dispose maintenant des moyens d'éliminer comme donneurs de sang tous les sujets qui présentent des stigmates sérologiques d'hépatite B actuelle ou passée.

D'autres formes d'hépatites, ne correspondant ni à une contagion alimentaire (hépatite A) ni au virus $B$, ont été décrites. On les a appelées provisoirement hépatites non-A nonB (NA-NB). C'est Chou, Américain d'origine chinoise, qui isola en 1982 un "nouveau" génome viral hépatotrope qu'il baptisa HBC. L'hépatite C est redoutable par son fréquent passage à la chronicité, avec risque de développement d'hépatome. Elle 
correspond à la grande majorité des hépatites NA-NB. Les autres, dont plusieurs agents viennent d'être identifiés [2, 3], se traduisent volontiers par une élévation du taux des transaminases, si bien que cet examen doit être pratiqué chez tout donneur de sang af in d'écarter nombre de sujets porteurs du virus.

A partir du début des années 1980, ce sont des infections rétrovirales qui ont été transmises par des transfusions de sang ou de dérivés sanguins: VIH, l'agent du SIDA et HTLV-1 et HTLV-2, agents de paraparésies spastiques tropicales et de lymphomes et leucémies humaines $\left(m / s n^{\circ} 3\right.$, vol. 2, p. 155) [4, 5]. Là encore, le dépistage sérologique permet aujourd'hui d'éliminer les donneurs infectés.

Reste à définir la transmissibilité éventuelle des prions par l'hémothérapie. Cette contamination est possible. Quelques formes sporadiques de maladie de Creutzfeldt-Jakob ont peut-être cette origine $\left(\mathrm{m} / \mathrm{s} n^{\circ} 6-7\right.$, vol. 10, p. 734). La transfusion sanguine semble responsable de certains cas décrits en Angleterre où sévit une épidémie massive chez les bovins: l'encéphalite spongiforme. Si les maladies liées aux prions sont dans notre espèce très exceptionnelles au moins à l'heure actuelle, il est difficile de prévoir l'avenir étant donné la lenteur de l'incubation et la quasi- inexistence de tests biologiques pour dépister les porteurs "sains" $(\mathrm{m} / \mathrm{s}$ $n^{\circ} 8$, vol. 11, p. 1160).

Les protéines plasmatiques peuvent aujourd'hui être fabriquées par génie génétique, le facteur VIII recombinant étant d'ores et déjà commercialisé. Des essais préliminaires de substituts du sang sont en cours transporteurs fluorocarbonés d'oxygène et de gaz carbonique, mais surtout hémoglobines réticulées [6]. Seules les plaquettes, du fait de leur complexité, demeureront longtemps irremplaçables et ne pourront être fournies que par des donneurs de sang.

Après le temps des triomphes et des certitudes, la transfusion sanguine a connu depuis trente ans bien des problèmes liés aux infections posttransfusionnelles, dont les conséquences ont été aggravées par une pratique peu prudente, culminant avec les «transfusions de confort».

Aujourd'hui, la sélection des donneurs, le dépistage, le traitement des produits, la précision des indications et, dans l'avenir, le recours croissant aux protéines recombinantes et aux substituts du sang aboutissent et aboutiront a des pratiques de moins en moins risquées... mais aussi de plus en plus chères... même pour les pays riches. Une transfusion sanguine sûre pour tous, voilà un nouveau défi à relever

\section{RÉFÉRENCES}

1. Ruffié J, Sournia JC. La Transfusion sanguine, Fayard, 1996.

2. Denis $F$, Nicot $T$. Découverte de nouveaux virus des hépatites, les « $\mathrm{GBV}$ »: quelle est leur place et quel est leur pouvoir pathogène? médecine/sciences 1995; 11 : 883-5.

3. Poisson F, Roingeard P, Goudeau A. Le virus de l'hépatite delta: un mode de réplication bien singulier. médecine/sciences 1995; 11: $1379-87$

4. Gessain A, de Thé G. Virus HTLV-I, leucémies $\mathrm{T}$ de l'adulte et neuromyélopathies chroniques. médecine/sciences $1987 ; 3$ : 471-8.

5. Koralnik I, Gessain A. Virus HTLV-I : structure et fonction des protéines de la région $\mathrm{pX}$. médecine/sciences 1994; 10 : 296 305 .

6. Pagnier J, Poyart C. Hémoglobine recombinante et transporteur d'oxygène artificiel. médecine/sciences $1992 ; 8: 790-6$.

\section{Jacques Ruffié}

Membre de l'Institut, professeur honoraire au Collège de France, 22, boulevard Flandrin, 75116 Paris, France.

\section{TIRÉS À PART}

J. Ruffié.

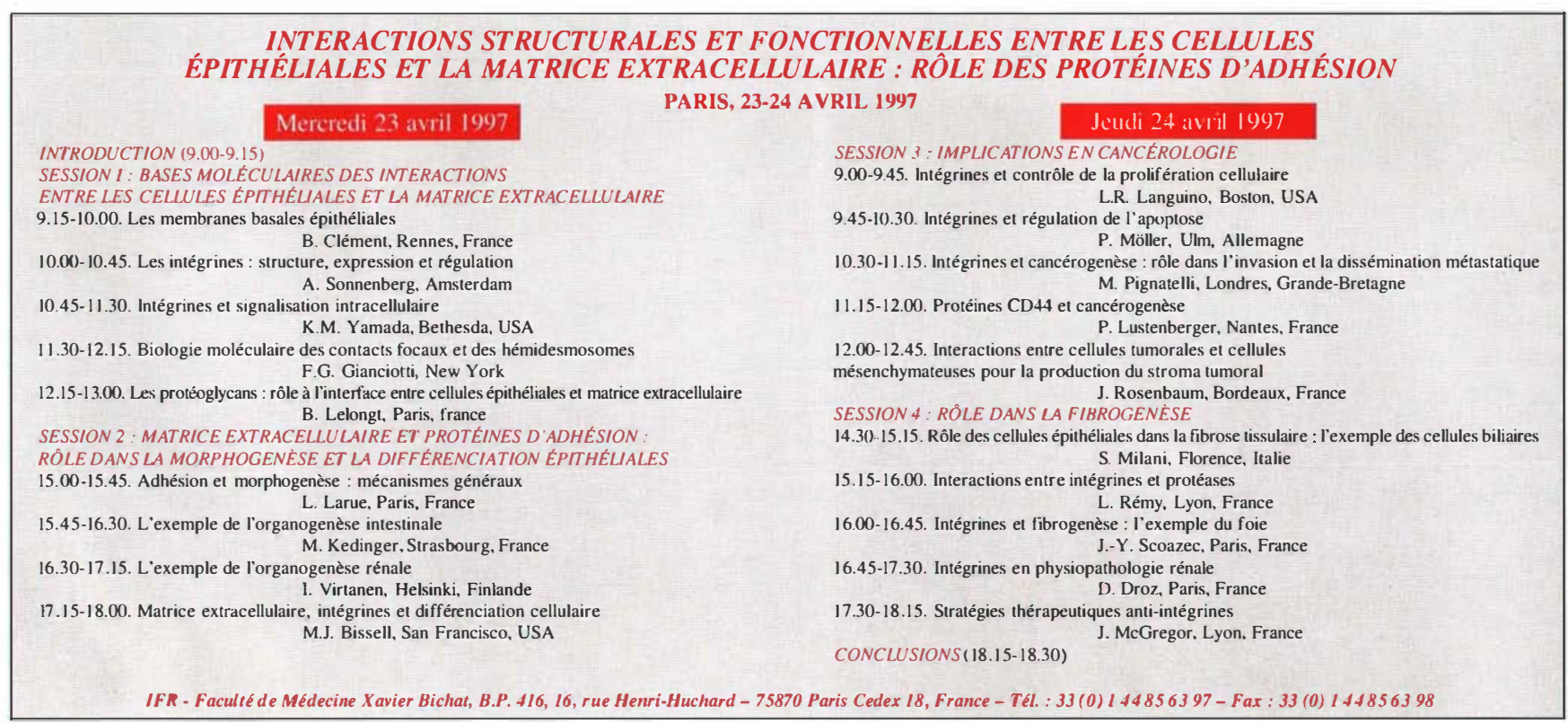

